\title{
Alteration in Pulmonary Blood Flow Induced by Cold Exposure to the Hand in Progressive Systemic Sclerosis
}

\author{
Kunihiko YAMAUCHI and Shigeru ARIMORI
}

\begin{abstract}
To determine whether pulmonary blood flow is altered by cold exposure to the hand, cold exposure was induced by immersion of the left hand in $7^{\circ} \mathrm{C}$ water in 8 patients with progressive systemic sclerosis (PSS). Lung perfusion was measured by continuous infusion of ${ }^{81} \mathrm{M}$ krypton into the right antecubital vein. Exposing the left hand to cold caused an 11 to $32 \%$ decrease in the pulmonary blood flow in all lung fields in 4 of 8 patients. After release of cold exposure, in one of the 4 patients demonstrating a decrease during cold exposure, pulmonary blood flow recovered, while it kept on a decrease in the remaining 3 patients. Physiological abnormalities of pulmonary blood flow in PSS have been shown as a quantitative change and a image.
\end{abstract}

Key Words: Perfusion lung scan, ${ }^{81} \mathrm{M}$ krypton

Pulmonary involvement with radiographic and/or lung function abnormalities has been observed in progressive systemic sclerosis (PSS) (1). Histological study of the parenchymal changes in the lungs of PSS patients reveal diffuse alveolar fibrosis and thicking (2). Vascular lesions consisting of intimal proliferation, medial hypertrophy and myxomatous changes are present in the pulmonary arterioles. With or without these changes, patients with PSS sometimes complain of dyspnea, palpitation and respiratory chest pain during cold exposure. While Raynaud's phenomenon classically has been thought to involve vasospasm of only the peripheral vasculature, more recent studies $(3,4)$ indicate that vital organs such as kidneys may also demonstrate vascular spasm. However, little is known about the changes in pulmonary blood flow that occur in patients with PSS during cold exposure.

Using continuous peripheral vein infusion of ${ }^{81} \mathrm{M}$ krypton, blood flow into lungs unaffected from the left ventricle can be clearly visualized due to efficient exhalation of ${ }^{81} \mathrm{M}$ krypton through the lungs and the ultrashort-lived isotope characteristics of ${ }^{81} \mathrm{M}$ krypton (5). This method offers considerable potential for serial non-invasive assessment of lung function.

This paper presents the results of studies in patients with PSS who had altered responses in pulmonary blood flow during and after cold exposure, as measured by this radionuclide technique.

\section{MATERIALS AND METHODS}

\section{Patients}

Eight patients with PSS were studied, all of whom met the 1980 preliminary criteria for diagnosis of PSS published by the American Rheumatism Association (6) (Table 1). These patients consisted of 6 females and 2 males with mean age of 57 years (range 45-62 years). The mean duration from onset of their first symptoms was 6.8 years (range 3-18 years). Cases 2, 3, 5, 7 and 8 were diagnosed as having pulmonary fibrosis by chest roentgenograms and pulmonary-function tests including a singlebreath diffusing capacity test for carbon monoxide (DLCO). Cases 1, 4 and 6 had no pulmonary fibrosis. Patients with bronchial asthma, pneumonia, cardiac failure and those who were cigarette smokers were also excluded.

From Department of Internal Medicine, Tokai University School of Medicine, Isehara

Received for publication December 20, 1988.

Reprint request to: Kunihiko Yamauchi, MD, Department of Internal Medicine,

Tokai University School of Medicine, Bohseidai, Isehara 259-11, Japan 
Table 1. Clinical characteristics and pulmonary functions in 8 patients with PSS.

\begin{tabular}{|c|c|c|c|c|c|c|c|c|c|c|}
\hline Case & Age/Sex & $\begin{array}{l}\text { Duration of digital } \\
\text { Raynaud's phenomenon } \\
\text { (year) }\end{array}$ & $\begin{array}{l}\text { Hemoglobin } \\
(\mathrm{g} / \mathrm{dl})\end{array}$ & $\begin{array}{c}\text { Creatinine } \\
(\mathrm{mg} / \mathrm{dl})\end{array}$ & $\begin{array}{l}\text { Pulmo } \\
\mathrm{VC}\end{array}$ & $\begin{array}{l}\text { nary funct } \\
\text { predicted } \\
F E V_{1.0}\end{array}$ & $\begin{array}{l}\text { on comp } \\
\text { normal } \\
T L C\end{array}$ & $\begin{array}{l}\text { ared with } \\
(\%) \\
\text { DLC }\end{array}$ & $\begin{array}{l}\text { Blood gas } \\
\mathrm{PaO}_{2}\end{array}$ & $\begin{array}{l}\text { Radiographic } \\
\text { pattern }\end{array}$ \\
\hline 1 & $55 / M$ & 4 & 13.5 & 1.0 & 96.6 & 89.6 & 92.3 & 95.6 & 92.6 & normal \\
\hline 2 & $62 / F$ & 12 & 9.8 & 0.8 & 79.3 & 92.0 & 102.2 & 52.5 & 85.3 & fibrosis \\
\hline 3 & $61 / F$ & 7 & 12.7 & 0.7 & 97.0 & 88.1 & 106.0 & 104.7 & 89.7 & fibrosis \\
\hline 4 & $58 / F$ & 1 & 9.0 & 0.9 & 97.6 & 75.5 & 100.1 & 139.8 & 73.8 & normal \\
\hline 5 & $55 / F$ & 6 & 15.3 & 0.9 & 101.9 & 77.0 & 66.7 & 32.1 & 80.7 & fibrosis \\
\hline 6 & $45 / F$ & 3 & 12.6 & 0.8 & 107.7 & 85.6 & 121.5 & 74.1 & 97.2 & normal \\
\hline 7 & $60 / F$ & 18 & 13.0 & 0.8 & 88.5 & 73.9 & 81.6 & 73.8 & 81.7 & fibrosis \\
\hline 8 & $62 / M$ & 7 & 14.7 & 1.1 & 83.7 & 74.6 & 74.5 & 49.4 & 93.8 & fibrosis \\
\hline
\end{tabular}

\section{Protocol}

Perfusion lung scans with ${ }^{81} \mathrm{M}$ krypton were performed after at least 2 hours in a warm room $\left(23^{\circ} \mathrm{C}\right)$. The subjects were placed in the supine position over a gamma camera with a high-resolution parallel-hole collimator linked to a dedicated minicomputer (PDP-11/34). All data acquisitions and analyses were performed using commercially available software. A generator of $370 \mathrm{MBq}$ containing ${ }^{81} \mathrm{M}$ kryipton gas, a product of ${ }^{81}$ rubidium, was used. The krypton was then administered in a $5 \%$ glucose solution by continuous infusion into the right antecubital vein at a rate of $8 \mathrm{ml} / \mathrm{min}$ using an infusion pump (Watson Marlow Ltd., Falmouth, Cornwall). After a steady state in the lungs was achieved by the infusion, ${ }^{81} \mathrm{M}$ krypton was infused during a control period of 2 to 3 minutes. Cold exposure was induced for 2 to 4 minutes by immersion of the left hand in $7^{\circ} \mathrm{C}$ water. After termination of cold exposure, recovery was observed for 2 or 3 minutes in a warm room $\left(23^{\circ} \mathrm{C}\right)$. Serial images at 5 -second intervals were collected on a magnetic disc. The total acquisition time for the test and intervention studies of each cold exposure was 6 to 8 minutes. Rectangular regions of interest (ROIs) were chosen in the each lung, avoiding the right cardiac area, and a time-activity curve at 10 -second intervals was generated from each ROI. However, curves of the left lung are mainly discussed, because time-activity curves of the right lung cannot mainly eliminate the influence of right cardiac activity. All patients studied here had digital Raynaud's phenomenon. Therefore, radioactive counts in lungs were normalized to remove a fluctuation of lung activity induced secondarily by a decrease of blood flow back to the right ventricle as a result of digital
Raynaud's phenomenon. Average counts of each $\mathrm{ROI}$ in the few minutes during and after cold exposure were compared with the counts before cold exposure.

\section{RESULTS}

During cold exposure to the left hand, 4 of 8 patients showed $11,18,28$ and $32 \%$ decreases, respectively, compared with $100 \%$ of before exposure. These pulmonary blood flows decreased within 10 seconds after the hand was immersed in cold exposure. In the remaining patients, the pulmonary blood flow did not fluctuate or caused an increase of less than $10 \%$. During 1 minute after termination of the cold exposure, in 3 of the 4 patients with decrease during cold exposure period was there partial return to the base line level in pulmonary blood flow because the extent of increase was small (Fig. 1). In all lung fields, pulmonary blood flow fluctuated in the same directions during and after cold exposure. Pulmonary fibrosis was revealed in 3 of 4 patients demonstrating a decrease during cold exposure period, while was not in the remaining one patient (case 6). In 2 of 4 patients with no change or mild increase in pulmonary blood flow during cold exposure was there pulmonary fibrosis, but not in the remaining two patients (cases $1 \& 4$ ). Decrease in pulmonary blood flow seems not to be associated with parenchymal lesions which are suggested by pulmonary-function tests and radiographic pattern (Table 1).

Pulmonary blood flow determination in case 7 , who was typical of this decrease in pulmonary blood flow, are shown in Figs. $2 a$ and $2 b$. 


\section{Lt. Hand}

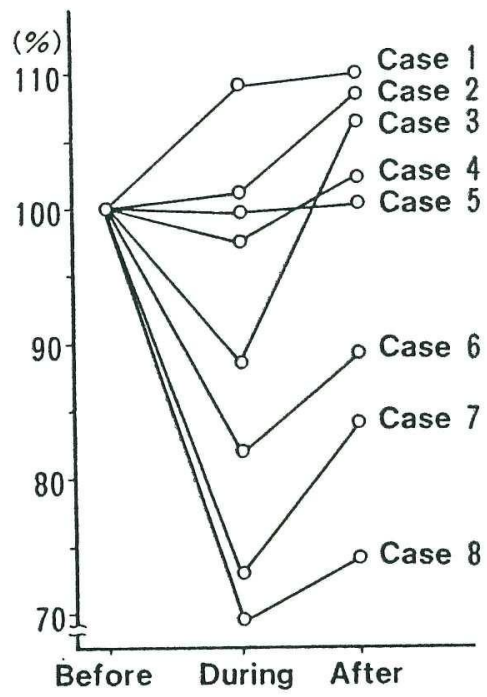

Fig. 1. Timed changes of pulmonary blood flow during and after cold exposure of the left hand, as compared with $100 \%$ flow before cold exposure. Each cycle means average counts of the left lung area a few minutes before, during and after cold exposure.

\section{DISCUSSION}

When ${ }^{81} \mathrm{M}$ krypton is infused continuously and a constant fraction of the ${ }^{81} \mathrm{M}$ krypton-labeled gas is removed by ventilation, the radioactivity in any lung region represents regional blood flow per unit of lung volume because its physical half-life of 13

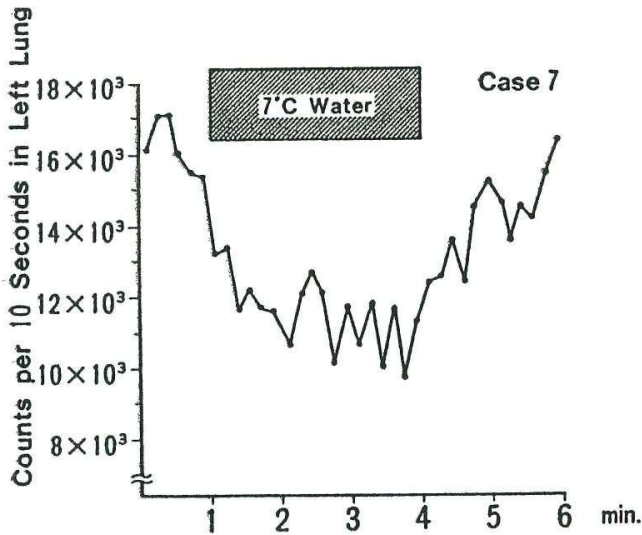

Fig. 2a. Time-radioactivity course of ${ }^{81} \mathrm{M}$ krypton lung perfusion scan in case 7. (-): left lung area counts in cold exposure to the left hand.
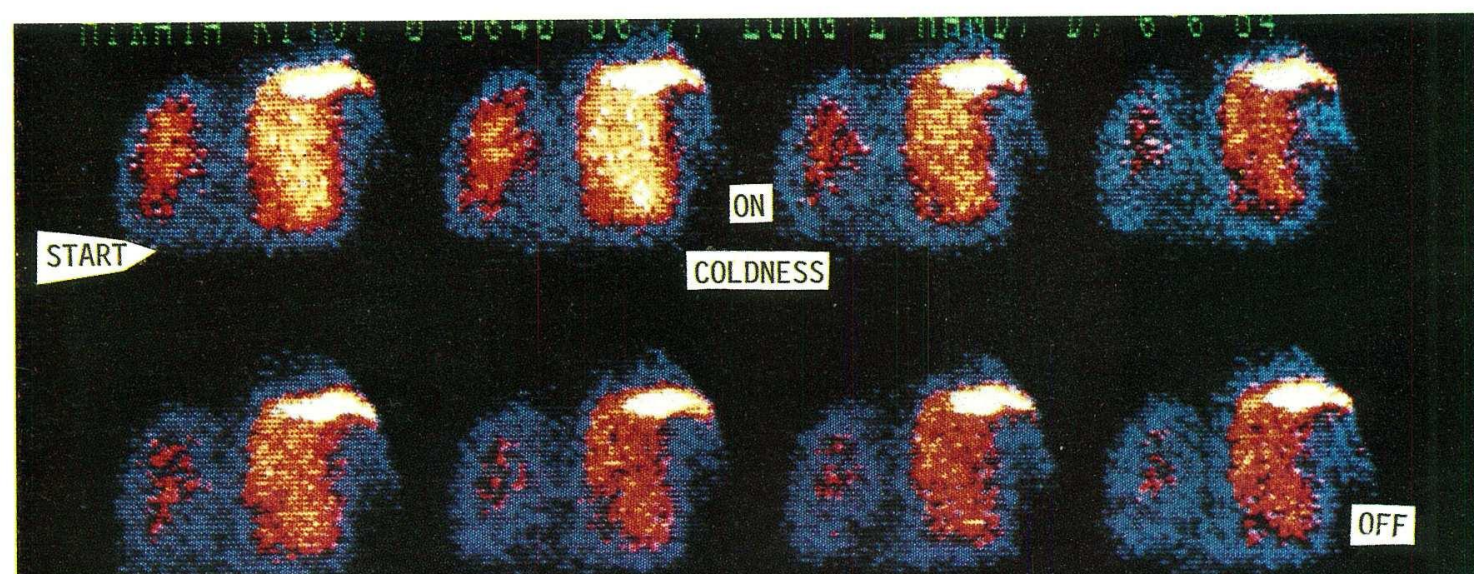

Fig. 2b. Posterior serial images at 30-second intervals during and after cold exposure to the left hand in case 7. 
seconds is much shorter than the expiratory time of an adult (5).

If the cold exposure makes the rate/depth of respiration increase, decrease in lung activity may result from rapid discharge of alveolar retention in ${ }^{81} \mathrm{M}$ krypton-labeled gas rather than decrease in pulmonary blood flow. In the present study the patients were allowed to breathe normally, under physiologic conditions and the cold stimulus to the left hand did not cause significant change in the rate/depth of the respiration. Therefore, a change in radioactive counts, which were normalized to remove the influence of digital Raynaud's phenomenon, reflects that in pulmonary blood flow.

To reveal abnormalities of pulmonary vascular dynamics in PSS patients, lung perfusion scan with ${ }^{81} \mathrm{M}$ krypton has been performed by Furst et al (7). They have revealed a decrease in pulmonary blood flow after immersion of the hand in ice water, judging from the images read blindly by 3 independent observers. It has been reported that pulmonary capillary vascular volume in PSS decreases during cold weather, suggesting increased pulmonary vascular tone during exposure to cold (8). In our investigation using krypton perfusion lung scan, decreased pulmonary blood flow in every field of lungs was demonstrated by continuous change in radioactivity and image during cold exposure of the left hand in 4 of 8 patients with PSS. Similar results as in PSS have been reported in most patients with systemic lupus erythematosus (SLE) (9). In a few patients with SLE, pulmonary blood flow has increased during cold exposure (9), while any PSS patients presented here did not reveal increase of pulmonary blood flow. In most patients with SLE who show decreased pulmonary blood flow with cold exposure, the flow has returned to the base line level within 1 minute after cessation of the cold stimulus (9). In contrast, in some patients with PSS, the decrease in pulmonary blood flow persisted at least for several minutes following the removal of the cold exposure. It may be the persistence of a "pulmonary Raynaud's phenomenon" that more likely leads to pulmonary fibrosis in patients with PSS, compared to patients with SLE. This means that, even if these patients are exposed to cold only for a few minutes, pulmonary vasospasm continues for a long time after the release of the cold. Therefore, pulmonary blood flow in PSS seems to be widely different from that in SLE.

Correlation between pulmonary fibrosis and the changes in pulmonary blood flow in PSS patients could not be found. This may mean that vascular lesions in the lungs of PSS patients are not parallel to the parenchymal changes.

Smooth muscle tone in the alveolar sacs and alveolar ducts has been reported to fail to respond to propranolol and isoproterenol in PSS patients, suggesting degenerative changes in the cholinergic and beta adrenergic receptors in addition to the loss of smooth muscle (8). Abnormal response of pulmonary blood flow to cold exposure also may reflect an abnormality in these receptors to the neurohumoral control of vascular smooth muscle fibers rather than parenchymal changes in the lungs.

\section{REFERENCES}

1) Sackner MA, Akgun N, Kimbel P, et al: The pathophysiology of scleroderma involving the heart and respiratory system. Ann Intern Med 60: 611, 1964.

2) Guttadauria M, Ellman H, Kaplan D: Progressive systemic sclerosis: Pulmonary involvement. Clin Rheum Dis 5: 151, 1979.

3) Cannon PJ, Hassar M, Case DB, et al: The relationship of hypertension and renal failure in scleroderma (progressive systemic sclerosis) to structural and functional abnormalities of the renal cortical circulation. Medicine 53: 1, 1974.

4) Kovalchik MT, Guggenheim SJ, Silverman MH, et al: The kidney in progressive systemic sclerosis. A prospective study. Ann Int Med 89: 881, 1978.

5) Ciofetta G, Pratt TA, Hughes JMB: Regional pulmonary perfusion assessed with continuous intravenous infusion of $\mathrm{Kr}-81 \mathrm{~m}$ : A comparison with Tc-99m macroaggregates. J Nucl Med 19: 1126, 1978.

6) Subcommittee for Scleroderma Criteria of the American Rheumatism Association Diagnostic and Therapeutic Criteria Committee. Preliminary criteria for the classification of systemic sclerosis (Scleroderma). Arthritis Rheum 23: 581, 1980.

7) Furst DE, Davis JA, Clements PJ, et al: Abnormalities of pulmonary vascular dynamics and inflammation in early progressive systemic sclerosis. Arthritis Rheum 24: 1403, 1981.

8) Emmanuel G, Saroja D, Gopinathan K, et al: Environmental factors and the diffusing capacity of the lung in progressive systemic sclerosis. Chest 69 (Suppl): 304, 1976.

9) Yamauchi K, Suzuki Y, Ichikawa Y, et al: Abnormalities of pulmonary blood flood during cold exposure in systemic lupus erythematosus. Nucl Med Comm 9: 423, 1988. 